Schließlich warfen Ingrid Kästner (Leipzig) und Susanne Hahn (Dresden) noch einen Blick in die Frühgeschichte medizinischer Moulagen, die nördlich der Alpen erstmals zu Beginn des 19. Jahrhunderts von Franz Heinrich Martens (1780-1805) und Wilhelm Gottlieb Tilesius (1769-1857) geschaffen wurden. Das kulinarische und das kulturelle Begleitprogramm im Stadtkern und in der landschaftlich attraktiven Umgebung Zürichs ließen keinen Frust aufkommen, arbeiten zu müssen, während andere Urlaub machen, sondern die an sich schon vergnügliche Arbeit (res severa verum gaudium) doppelt zur Lust werden, wofür den Veranstaltem in Zürich auch an dieser Stelle nochmals herzlich gedankt sei.

Susanne Hahn (Dresden)

\title{
Bericht vom Deutschen Wissenschaftshistoriker- tag 26. bis 29. September 1996 in Berlin
}

Traditionell ist der Monat September reich an wissenschaftlichen Jahrestagungen. Im September 1996 tagten die Historiker in München, die Philosophen in Leipzig, die Mathematiker (mit mathematikhistorischer Fachsektion) in Jena und so ließe sich die Reihe fortsetzen. Neu in diesen Reigen deutscher wissenschaftlicher Veranstaltungen trat erstmals ein Wissenschaftshistorikertag, organisiert von einem Verein zur Durchführung des deutschen Wissenschaftshistorikertages 1996 in Berlin e.V. unter Vorsitz von Rüdiger vom Bruch, Professor für Wissenschaftsgeschichte an der Humboldt-Universităt Berlin. Diese organisatorische Neuheit in der deutschen Wissenschaftslandschaft wurde von dem Beschluß der Gesellschaft für Wissenschaftgeschichte, der Deutschen Gesellschaft für Geschichte der Međizin, Naturwissenschaft und Technik sowie der Georg-Agricola-Gesellschaft getragen, erstmals ihre Jahrestagungen am selben Ort, zur selben Zeit und zum selben Thema durchzuführen.

Eine Reihe weiterer Gremien und Institutionen schlossen sich mit eigenen Symposien an, so die Gesellschaft für Wissenschafts- und Universitätsgeschichte, das Archiv des naturhistorischen Museums Wien, die Herzog-August-Bibliothek Wolfenbüttel, die Internationale Gesellschaft zur Erforschung der Salzgeschichte, die Julius-Hirschberg-Gesellschaft (deutschsprachige Vereinigung für Geschichte der Augenheilkunde), die Deutsche Forschungsgemeinschaft, die Gesellschaft zur Förderung der Religion/Umwelt-Forschung e.V., die Deutsche Gesellschaft für Geschichte der Pharmazie, die Arbeitsgruppe Geschichte der Meteorologie der Deutschen Meteorologischen Gesellschaft, der Arbeitskreis Geschichte der Polarforschung der Deutschen Gesellschaft für Polarforschung, die Berliner Gesellschaft für Geschichte der Medizin, die Fachgruppe Geschichte der Chemie der Gesellschaft Deutscher Chemiker, der Fachverband Physikgeschichte der Deutschen Physikalischen Gesellschaft, der Arbeitskreis Geschichte der Meeresforschung der Deutschen Gesellschaft für Meeresforschung e.V., der Arbeitskreis für Geschichte der Germanistik, die Karl-Lamprecht-Gesellschaft Leipzig e.V., die Fachgruppe Geschichte der Psychologie der Deutschen Gesellschaft für Psychologie, der Arbeitskreis Geschichte der Geowissenschaften, die Deutsche Gesellschaft für Geschichte und Theorie der Biologie e.V., das Osteuropa-Institut der Freien Universität Berlin. Das 1995 in Berlin gegründete Max-PlanckInstitut für Wissenschaftsgeschichte beteiligte sich mit herausgehobenen Vorträgen durch ihre Direktoren sowie mit Beiträgen zahlreicher wissenschaftlicher Angehöriger des Instituts.

Das Thema „Zeitenwenden, Neuorientierungen in Wissenschaft und Gesellschaft", wobei die Einschnitte um 1600 und um 1900 zum Mittelpunkt erklärt worden waren, wurde somit auf interessante Weise von verschiedenen Seiten beleuchtet. 400 angemeldete Teilnehmer sowie 500 bis 600 tatsächliche Besucher, wie Herr vom Bruch am Ende des Kongresses mitteilte, trafen auf ein breites Spektrum von Vorträgen. Die Fülle von Parallelveranstaltungen ermöglichte es dem einzelnen nicht, die Gesamtheit des Angebots zu überschauen. Die mit der Zeit um 1900 befaßten Vorträge sind für einen gemeinsamen Band vorgesehen, der im Nomos-Verlag erscheinen soll. NTM bringt einige bei der Redaktion eingegangene Berichte über einzelne Sitzungen des Wissenschaftshistorikertages in loser Folge.

R. Tobies (Berlin) 